\title{
A study of metabolic syndrome in women with polycystic ovary syndrome at tertiary care center
}

\author{
Chelsae Kuntal*, Jyotsna Vyas, Asha Chaudhary, Sunita Hemani, Lata Rajoria
}

Department of Obstetrics and gynaecology, SMS Medical College Jaipur, Rajasthan, India

Received: 07 April 2021

Accepted: 04 May 2021

*Correspondence:

Dr. Chelsae Kuntal,

E-mail: chelsaekunta16944@gmail.com

Copyright: (C) the author(s), publisher and licensee Medip Academy. This is an open-access article distributed under the terms of the Creative Commons Attribution Non-Commercial License, which permits unrestricted non-commercial use, distribution, and reproduction in any medium, provided the original work is properly cited.

\begin{abstract}
Background: Polycystic ovary syndrome is a common endocrinopathy in women of reproductive age with prevalence of $6-10 \%$ which is characterized by hyper androgenic features and chronic oligo - anovulation and polycystic ovary morphology. Most women with polycystic ovary syndrome are also characterized by metabolic abnormalities like insulin resistance, hyperinsulinemia, dyslipidemia and abdominal obesity, these forming risk factors for metabolic syndrome. The objective of the study was to compare the clinical, biochemical and hormonal profile of polycystic ovary syndrome patients with and without metabolic syndrome.

Methods: A comparative cross- sectional study was undertaken on 79 PCOS women diagnosed with PCOS according to Rotterdam criteria, in which the clinical data and hormonal profile of two groups of polycystic ovary syndrome women with and without metabolic syndrome was compared.

Results: The mean age of 79 patients in this study group with and without metabolic syndrome was $26.17 \pm 3.18$ and $25.57 \pm 3.41$ years respectively. There were more patients from urban areas as compared to rural areas and maximum patients. Significantly higher number of PCOS women with metabolic syndrome had hirsutism and acanthosis nigricans than those without metabolic syndrome. Mean value of Waist circumference, systolic BP pressure, diastolic BP, S. Triglyceride and fasting glucose were higher and HDL levels were lower in women with metabolic syndrome than those without metabolic syndrome. Fasting insulin and HOMA-IR values were significantly higher in PCOS women with metabolic syndrome in comparison to those without metabolic syndrome.

Conclusion: PCOS is not only is the most frequent cause of anovulation, but it is also associated with characteristic metabolic disturbances that may have important implications for the long term health. Metabolic syndrome is a cluster of endocrine disturbances, including insulin resistance, dyslipidemia, obesity, and hypertension. It is associated with a two-fold increased risk of cardiovascular disease and a five-fold increased risk of type 2 diabetes. This illustrates the importance of early detection of insulin resistance and metabolic syndrome with subsequent application of preventive measures in women with polycystic ovary syndrome
\end{abstract}

Keywords: Polycystic ovary syndrome, Insulin resistance, Metabolic syndrome

\section{INTRODUCTION}

Polycystic ovary syndrome is a common endocrinopathy in women of reproductive age with prevalence of 6-10\% which is characterized by hyper androgenic features and chronic oligo - anovulation and polycystic ovary morphology. ${ }^{1-3}$ The criteria currently used for diagnosis of
PCOS are those established by the Rotterdam consensus which defines the disease as the presence of two of the three following criteria:- Oligomenorrhoea and/or anovulation, clinical and/or biochemical signs of hyperandrogenism and polycystic ovaries on ultrasound (presence of 12 or more follicles measuring 2-9 $\mathrm{mm}$ in diameter or with a total volume more than $10 \mathrm{~cm} 3$ in at 
least one ovary). Most women with polycystic ovary syndrome are also characterized by metabolic abnormalities like insulin resistance, hyperinsulinemia, dyslipidemia and abdominal obesity, these forming risk factors for metabolic syndrome. ${ }^{5}$

Metabolic syndrome (syndrome $\mathrm{X}$ ) is a cluster of metabolic disorders that confer increased risk of cardiovascular disease and diabetes mellitus. Thus metabolic syndrome is a major challenge to health care system. Women with polycystic ovary syndrome are at greater risk because they are exposed to risk factors at young age. ${ }^{6}$ Metabolic syndrome is assessed using NCEP ATP III criteria, if three or more of the following are satisfied: ${ }^{7}$

Waist circumference $\geq 80 \mathrm{~cm}$ (in Asian women). Triglyceride $150 \mathrm{mg} / \mathrm{dl}$. Systolic BP $\geq 130 \mathrm{mmHg}$, Diastolic BP $\geq 85 \mathrm{mmHg}$. HDL $\leq 50 \mathrm{mg} / \mathrm{dl}$. Fasting glucose $\geq 100 \mathrm{mg} / \mathrm{dl}$.

In present study, our aim is to compare clinical, biochemical and hormonal profile of PCOS women with and without metabolic syndrome so that we could evaluate metabolic syndrome in PCOS women at the earliest.

\section{METHODS}

This comparative cross-sectional study was conducted in the Department of obstetrics and gynaecology in SMS medical college, Jaipur between May 2019 to May 2020 with approval from the institute's ethics committee.

The study was carried out on 79 patients diagnosed with PCOS, according to the criteria of Rotterdam ESHRE/ASRM PCOS group's revised 2003 criteria. Individuals with specific disorders, such as adult- onset congenital adrenal hyperplasia, hyperprolactinemia, and androgen secreting neoplasia were excluded from the study. Patients taking medications example- steroids, oral contraceptives, metformin, or thiazide diuretics before starting the study were excluded.

All anthropometric measurements were done following standard protocol and calibrated instruments. Waist circumference was measured in centimetres on bare skin at the narrowest indentation between the 10th rib and the iliac crest at mid- respiration, in standing position and hip circumference was measured at the utmost circumference with undergarment .Height was measured without footwear against a well - fixed tape. Weight was measured in light clothing without footwear on a platform weighing scale. The Body mass index (BMI) was calculated as body weight (in $\mathrm{kg}$ ) divided by square of height (in metre). Blood pressure was recorded by a mercury sphygmomanometer twice after the subject was resting in a sitting position for 5 minutes, in left arm with appropriate size cuff by auscultatory method. The average of the two measurements was taken as the subject's blood pressure. Hirsutism was assessed using Modified Ferriman - gallwey score. In Ferriman- gallwey score, nine body areas are assessed and scored from 0-4. Increasing score correspond to greater density within a given area. Score $>/=8$ is used to define hirsutism.

\section{Sample collection and estimation}

After an overnight fast of at least $8 \mathrm{hrs}$, a venous blood sample was obtained from each subject and following investigations were done : fasting glucose estimation, ELISA for insulin level estimation (in micro U/ml), S. Testosterone, TSH (to rule out hypothyroidism), prolactin and lipid profile (S. cholesterol, triglyceride, HDL cholesterol levels) using enzymatic colorimetric technique. Insulin resistance was assessed using the Homeostatic model assessment -Insulin resistance (HOMA-IR) formula.

$$
\begin{aligned}
H O M A-I R=\text { fasting insulin }\left(\frac{u U}{m L}\right) & \\
& \times \text { fasting glucose }\left(\frac{m g}{d L}\right) \div 405
\end{aligned}
$$

Insulin resistance was defined by HOMA-IR value $\geq 2.5$.

Abdominal / transvaginal sonography was performed in early follicular phase in radiology department of SMS hospital, Jaipur. Polycystic ovary was defined as $\geq 12$ follicles per ovary that are $2-9 \mathrm{~mm}$ in diameter and/ or ovarian volume $>10 \mathrm{~cm} 3$.

Metabolic syndrome was assessed using NCEP ATP III criteria, if three or more of the following are satisfied: ${ }^{7}$ Waist circumference $\geq 80 \mathrm{~cm}$ (in Asian women). Triglyceride $150 \mathrm{mg} / \mathrm{dl}$. Systolic BP $\geq 130 \mathrm{mmHg}$, Diastolic $\quad \mathrm{BP} \geq 85 \mathrm{mmHg}$. HDL $\leq 50 \mathrm{mg} / \mathrm{dl}$. Fasting glucose $\geq 100 \mathrm{mg} / \mathrm{dl}$.

\section{Statistical analysis}

Continuous variables were summarized as mean and standard deviation while nominal/categorical variables were expressed as percentages. Unpaired t-test was used for analysis of continuous variables while chi-square test and Fischer Exact test were used for nominal/categorical variable. Sensitivity, specificity PPV and NPV were calculated using standard formulae. p-value $<0.05$ was taken as significant. Medcalc 16.4 version software was used for all statistical calculations.

\section{RESULTS}

The mean age of 79 PCOS women with and without metabolic syndrome was $26.17 \pm 3.18$ and $25.57 \pm 3.41$ years respectively .There were more patients from urban areas as compared to rural areas, $60.87 \%$ PCOS women without metabolic syndrome and $54.55 \%$ women with metabolic syndrome belonged to urban areas (Table 1). 
Maximum patients were null gravida in both the groups. Among PCOS women without metabolic syndrome, $65 \%$ women have irregular cycles and $64 \%$ PCOS women with metabolic syndrome have irregular cycle frequency. Significantly higher number of PCOS women with metabolic syndrome had hirsutism $(41.3 \%$ versus $81.8 \%)$ and acanthosis nigricans (19.6\% versus $51.5 \%)$ than those without metabolic syndrome. (Table 2). Mean value of waist circumference $(91.38 \pm 8.30$ versus $78.35 \pm 4.36)$, systolic blood pressure $(122.06 \pm 11.99$ versus $114.35 \pm 9.10)$, diastolic blood pressure $(79.85 \pm 8.88$ versus $74.35 \pm 9.10), \quad$ S. Triglyceride $(1.547 \pm 0.536$ versus $1.078 \pm 0.273)$, and fasting glucose $(98.25 \pm 13.94$ versus $84.37 \pm 9.07)$ was higher in PCOS women with metabolic syndrome in comparison to those without metabolic syndrome and HDL levels were lower in women with metabolic syndrome than those without metabolic syndrome $(41.19 \pm 4.88$ versus $43.85 \pm 6.43)$.

Table 1: Demographic factors in PCOS women with and without metabolic syndrome.

\begin{tabular}{|c|c|c|c|c|}
\hline & & $\begin{array}{l}\text { PCOS women without } \\
\text { metabolic syndrome }(n=46)\end{array}$ & $\begin{array}{l}\text { PCOS women with } \\
\text { metabolic syndrome } \\
(n=33)\end{array}$ & p value \\
\hline Mean Age (in years) & & $25.57 \pm 3.41$ & $26.17 \pm 3.18$ & 0.08 \\
\hline \multirow{2}{*}{ Residence } & Rural & $39.1 \%$ & $45.5 \%$ & \multirow{2}{*}{0.57} \\
\hline & Urban & $60.9 \%$ & $54.5 \%$ & \\
\hline \multirow[t]{5}{*}{ Socioeconomic status } & Class I & 0 & $3.03 \%$ & 0.87 \\
\hline & Class II & $47.83 \%$ & $42.42 \%$ & 0.87 \\
\hline & Class III & $52.17 \%$ & $54.55 \%$ & 0.87 \\
\hline & Class IV & 0 & 0 & 0.87 \\
\hline & Class V & 0 & 0 & 0.87 \\
\hline \multirow[t]{2}{*}{ Religion } & Hindu & $91.3 \%$ & $87.88 \%$ & 0.71 \\
\hline & Muslim & $8.7 \%$ & $12.12 \%$ & 0.71 \\
\hline
\end{tabular}

Table 2: Clinical Parameters in PCOS women with and without metabolic syndrome.

\begin{tabular}{|c|c|c|c|c|c|c|}
\hline & & \multicolumn{2}{|c|}{$\begin{array}{l}\text { PCOS without metabolic } \\
\text { syndrome }(n=46)\end{array}$} & \multicolumn{2}{|c|}{$\begin{array}{l}\text { PCOS with metabolic } \\
\text { syndrome }(n=33)\end{array}$} & \multirow[t]{2}{*}{ P value } \\
\hline & & No. & $\%$ & No. & $\%$ & \\
\hline \multirow{2}{*}{ Menstrual history } & Regular & 16 & 34.78 & 12 & 36.36 & \multirow[b]{2}{*}{0.88} \\
\hline & Irregular & 30 & 65.22 & 21 & 63.64 & \\
\hline \multirow{3}{*}{$\begin{array}{l}\text { Obstetrics } \\
\text { history }\end{array}$} & Null gravida & 26 & 56.52 & 17 & 51.52 & \multirow[b]{3}{*}{0.47} \\
\hline & Primigravida & 10 & 21.74 & 5 & 15.15 & \\
\hline & Multigravida & 10 & 21.74 & 11 & 33.33 & \\
\hline \multicolumn{2}{|l|}{ Hirsutism } & 19 & 41.3 & 27 & 81.8 & $<0.001$ \\
\hline \multicolumn{2}{|l|}{ Acne } & 14 & 30.4 & 11 & 33.3 & 0.78 \\
\hline \multicolumn{2}{|c|}{ Acanthosis Nigricans } & 9 & 19.6 & 17 & 51.5 & $<0.001$ \\
\hline
\end{tabular}

Table 3: Comparison of determinants of metabolic syndrome in PCOS women with and without metabolic syndrome.

\begin{tabular}{|c|c|c|c|}
\hline & $\begin{array}{l}\text { PCOS } \\
\text { Without MetS }(n=46)\end{array}$ & $\begin{array}{l}\text { PCOS } \\
\text { with MetS }(n=33)\end{array}$ & P value \\
\hline Waist circumference $(\mathrm{cm})$ & $78.35 \pm 4.36$ & $91.38 \pm 8.30$ & $<0.001$ \\
\hline Systolic blood pressure (mmHg) & $114.35 \pm 9.10$ & $122.06 \pm 11.99$ & $<0.01$ \\
\hline Diastolic blood pressure (mmHg) & $74.35 \pm 9.10$ & $79.85 \pm 8.88$ & $<0.01$ \\
\hline Triglyceride (mmol /L) & $1.078 \pm 0.273$ & $1.547 \pm 0.536$ & $<0.001$ \\
\hline HDL (mg /dl) & $43.85 \pm 6.43$ & $41.19 \pm 4.88$ & 0.04 \\
\hline Fasting glucose (mg/ dl ) & $84.37 \pm 9.07$ & $98.25 \pm 13.94$ & $<0.001$ \\
\hline
\end{tabular}

Table 4: Comparison of markers of insulin resistance in PCOS women with and without metabolic syndrome.

\begin{tabular}{|llll|} 
& $\begin{array}{l}\text { PCOS Without Metabolic } \\
\text { Syndrome }(\mathbf{n = 4 6})\end{array}$ & $\begin{array}{l}\text { PCOS With Metabolic } \\
\text { Syndrome }(\mathbf{n = 3 3})\end{array}$ & P \\
\hline Fasting Insulin & $15.17 \pm 10.21$ & $20.38 \pm 8.68$ & $<0.01$ \\
\hline HOMA-IR & $1.92 \pm 0.88$ & $6.47 \pm 1.88$ & $<0.001$ \\
\hline
\end{tabular}


There was significant difference in systolic and diastolic blood pressure, waist circumference, triglyceride, HDL and fasting plasma glucose between PCOS women with and without metabolic syndrome ( $\mathrm{p}$ value < .05) (Table 3$)$.

Fasting insulin $(20.38 \pm 8.68$ versus $15.17 \pm 10.21)$ and HOMA-IR values $(6.47 \pm 1.88$ versus $1.92 \pm 0.88)$ were significantly higher in PCOS women with metabolic syndrome in comparison to those without metabolic syndrome (Table 4).

\section{DISCUSSION}

Polycystic ovary syndrome is a common endocrinopathy in women of reproductive age with prevalence of 6-10\%, which is characterized by hyper androgenic features and chronic oligo - anovulation and polycystic ovary morphology. ${ }^{1-3}$ This study was undertaken to study the broad range of clinical, biochemical and hormonal features associated with this syndrome.

As PCOS is proposed to be more prevalent in younger age group than older women due to physiological decline in follicular cohort leading to a normalized ovarian ultrasonographic appearance with advancing age. In our study, maximum patients in both groups were aged between 21- 30 years. Mean age of PCOS women without metabolic syndrome was $25.57 \pm 3.41$ years whereas mean age of PCOS women with metabolic syndrome was $26.17 \pm 3.18$ years. There was no statistically significant difference in age in both the groups ( $\mathrm{p}$ value 0.08 ). These results were almost similar to study done by Varghese et al (2015) in which no association was found between age distribution of PCOS cases with development of metabolic syndrome ( $\mathrm{p}$ value- 0.12$).{ }^{8}$ According to the study conducted by Xiang et al (2013), women with and without metabolic syndrome $(25.2 \pm 5.1$ versus $24.6 \pm 4.6)$ were both similar in age ( $\mathrm{p}$ value 0.478$){ }^{9}$

In our study, more women in both groups belonged to urban areas, $60.87 \%$ PCOS women without metabolic syndrome and $54.55 \%$ women with metabolic syndrome belonged to urban areas. The less percentage of rural patients may be due to difference in catchment area of hospital or due to lack of awareness. High incidence of PCOS cases in urban areas could also be attributed to sedentary lifestyle and food habits acting as risk factors of PCOS. Among PCOS women without metabolic syndrome $91.30 \%$ belonged to Hindu religion $8.70 \%$ belonged to Muslim religion. Among PCOS women with metabolic syndrome, $87.88 \%$ belonged to Hindu religion and $12.12 \%$ belonged to Muslim religion. There was no statistically significant difference between both the groups on the basis of religion. This reflects the demographic characteristics in the catchment area of the hospital.

According to kuppuswamy's socio-economic classification, maximum number of women in both groups belonged to Class-II (upper-middle class) and Class-III (lower-middleclass) and 1 PCOS women with metabolic syndrome belonged to Class-I (upper class). There were no patients from Class-IV and Class-V. In PCOS women without metabolic syndrome, $65.22 \%$ women had irregular cycles and $34.78 \%$ had regular cycles and $63.64 \%$ PCOS women with metabolic syndrome had irregular cycle frequency and $36.36 \%$ PCOS women had regular menstrual cycles. There was no statistically significant difference between both groups. These results were similar to study conducted by Christodoulopoulou et al (2016) in which there was no significant difference between the hormonal and metabolic profile of PCOS women with and without menstrual cycle abnormalities. maximum numbers of patients were null gravida in both the groups. There was no statistically significant difference in both the groups on the basis of obstetric history. ${ }^{10}$

Significantly higher number of PCOS women had hirsutism (41.3\% versus $81.8 \%$ ) and acanthosis nigricans (19.6\% versus $51.5 \%)$ in those with metabolic syndrome than in whom metabolic syndrome was not present. This was similar to study conducted Mandrelle et al (2012) in which hirsutism (42.22\% versus 20\%) and acanthosis nigricans $(28.8 \%$ versus $8 \%$ ) were seen more in PCOS women with metabolic syndrome as compared to those without metabolic syndrome. Acne was seen in $30.4 \%$ PCOS women with metabolic syndrome compared to $33.3 \%$ cases without metabolic syndrome. There was no statistically significant difference between both the groups. Mean value of waist circumference $(91.38 \pm 8.30$ versus $78.35 \pm 4.36)$, systolic blood pressure (122.06 \pm 11.99 versus $114.35 \pm 9.10)$, diastolic blood pressure $(79.85 \pm 8.88$ versus $74.35 \pm 9.10), \mathrm{S}$. Triglyceride $(1.547 \pm 0.536$ versus $1.078 \pm 0.273)$, and fasting glucose $(98.25 \pm 13.94$ versus $84.37 \pm 9.07)$ was higher in PCOS women with metabolic syndrome in comparison to those without metabolic syndrome and HDL levels were lower in women with metabolic syndrome than those without metabolic syndrome $(41.19 \pm 4.88$ versus $43.85 \pm 6.43)$. There was significant difference in systolic and diastolic blood pressure, waist circumference, triglyceride, HDL and fasting plasma glucose between PCOS women with and without metabolic syndrome ( $p$ value $<0.05$ ). This was similar to study conducted by Xiang et al (2013) in which, PCOS women with metabolic syndrome had significantly increased BMI, WC, SBP, DBP, fasting blood glucose and triglyceride compared to those without metabolic syndrome ( $\mathrm{p}$ value $<0.001$ ) and HDL cholesterol levels were significantly lower in women with metabolic syndrome. ${ }^{9} \quad$ Fasting insulin $(20.38 \pm 8.68$ verus $15.17 \pm 10.21)$ and HOMA-IR values $(6.47 \pm 1.88$ versus $1.92 \pm 0.88)$ were significantly higher in PCOS women with metabolic syndrome in comparison to those without metabolic syndrome.

\section{CONCLUSION}

PCOS is not only is the most frequent cause of anovulation, but it is also associated with characteristic metabolic disturbances that may have important implications for the long term health. Metabolic syndrome 
is a cluster of endocrine disturbances, including insulin resistance, dyslipidemia, obesity, and hypertension. It is associated with a two-fold increased risk of cardiovascular disease and a five-fold increased risk of type 2 diabetes. This illustrates the importance of early detection of insulin resistance and metabolic syndrome with subsequent application of preventive measures in women with polycystic ovary syndrome.

Funding: No funding sources Conflict of interest: None declared

Ethical approval: The study was approved by the Institutional Ethics Committee

\section{REFERENCES}

1. Knochenhauer ES, Key TJ, Kahsar-Miller M, Waggoner W, Boots LR, Azziz R. Prevalence of the polycystic ovary syndrome in unselected black and white women of the south eastern United States: a prospective study. J Clin Endocrinol Metab. 1998;83:3078-82.

2. Diamanti-Kandarakis E, Kouli CR, Bergiele AT. A survey of the polycystic ovary syndrome in the Greek island of Lesbos: hormonal and metabolic profile. J Clin Endocrinol Metab. 1999;84:4006-11.

3. Azziz R, Woods KS, Reyna R, Key TJ, Knochenhauer ES, Yildiz BO. The prevalence and features of the polycystic ovary syndrome in an unselected population. J Clin Endocrinol Metab. 2004;89:27459.

4. Rotterdam ESHRE/ASRM-Sponsored PCOS Consensus Workshop Group. Revised 2003 consensus on diagnostic criteria and long term health risks related to polycystic ovary syndrome. Fertil Steril. 2004;81(1):19-25.

5. Diamanti-Kandarakis E, Dunaif A. Insulin resistance and polycystic ovary syndrome revisited : an update on mechanisms \& implications. Endocr Rev. 2012;33:981-1030.

6. Rossi B, Sukalich S, Droz J. Prevalence of metabolic syndrome and related characteristics in obese adolescents with and without polycystic ovary syndrome. J Clin Endocrinol Metab. 2008;93:4780-6.

7. National Cholesterol Education Program (NCEP). Expert panel on detection, evaluation and treatment of high blood cholesterol in adult (Adult Treatment Panel III), "Third Report of the National Cholesterol Education Program (NCEP) Expert Panel on Detection, Evaluation \& Treatment of High Blood Cholesterol in Adults (Adult Treatment Panel III) Final Report. Circulation. 2002;106:3143-421.

8. Varghese J, Kantharaju S, Thunga S, Joseph N, Singh PK. Prevalence and predictors of metabolic syndrome in women with polycystic ovarian syndrome: a study from Southern India. Int J Reprod Contracept Obstet Gynecol. 2015;4(1):113-8.

9. Xiang S, Hua F, Chen L, Tang Y, Jiang X, Liu Z. Lipid accumulation product is related to metabolic syndrome in women with polycystic ovary syndrome. Exp Clin Endocrinol Diabetes. 2013;121(2):115-8.

10. Christodoulopoulou V, Trakakis E, Pergialiotis V, Peppa M, Chrelias C, Kassanos D and Papantoniou N. Clinical and Biochemical Characteristics in PCOS Women With Menstrual Abnormalities. J Family Reprod Health. 2016;10(4):184-90.

11. Mandrelle K, Kamath MS, Bondu DJ, Chandy A, Aleyamma TK and George K. Prevalence of metabolic syndrome in women with polycystic ovary syndrome attending an infertility clinic in a tertiary care hospital in south India. J Hum Reprod Sci. 2012;5(1):26-31.

Cite this article as: Kuntal C, Vyas J, Chaudhary A, Hemani S, Rajoria L. A study of metabolic syndrome in women with polycystic ovary syndrome at tertiary care center. Int J Reprod Contracept Obstet Gynecol 2021;10:2427-31. 